\title{
Ultraclean Two-Stage Aerosol Reactor for Production of Oxide-Passivated Silicon Nanoparticles for Novel Memory Devices
}

\author{
Michele L. Ostraat, ${ }^{a}$ Jan W. De Blauwe, ${ }^{b}$ Martin L. Green, ${ }^{b, *}$ L. Douglas Bell, ${ }^{c}$ \\ Harry A. Atwater, and Richard C. Flagan ${ }^{\mathrm{a}}$ \\ ${ }^{a}$ California Institute of Technology, Pasadena, California 91125, USA \\ ${ }^{b}$ Agere Systems, Murray Hill, New Jersey 07974, USA \\ ${ }^{c} J e t$ Propulsion Laboratory, Pasadena, California 91109, USA
}

\begin{abstract}
Silicon nanoparticle-based floating gate metal oxide semiconductor field effect devices are attractive candidates for terabit $\mathrm{cm}^{-2}$ density nonvolatile memory applications. We have designed an ultraclean two-stage aerosol process reactor and $200 \mathrm{~mm}$ wafer deposition chamber in order to integrate $\mathrm{Si} / \mathrm{SiO}_{2}$ nanoparticles into memory devices. In the first stage, silicon nanoparticles are synthesized by thermal decomposition of silane gas in a reactor that has been optimized to produce nonagglomerated nanoparticles at rates sufficient for layer deposition. In the second stage, the silicon particles are passivated with thermal oxide that partly consumes the particle. This two-stage aerosol reactor has been integrated to a $200 \mathrm{~mm}$ silicon wafer deposition chamber that is contained within a class 100 cleanroom environment. This entire reactor system conforms to rigorous cleanliness specifications such that we can control transition metal contamination to as good as $10^{10}$ atoms $\mathrm{cm}^{-2}$. The deposition chamber has been designed to produce a controllable particle density profile along a $200 \mathrm{~mm}$ wafer where particles are thermophoretically deposited uniformly over three-quarters of the wafer. Thus, we now have the capability to deposit controlled densities of oxide-passivated silicon nanoparticles onto $200 \mathrm{~mm}$ silicon wafers for production of silicon nanoparticle memory devices.

(C) 2001 The Electrochemical Society. [DOI: 10.1149/1.1360210] All rights reserved.
\end{abstract}

Manuscript received October 4, 2000.

Nonvolatile memory devices where the floating gate consists of a dense array of embedded silicon nanoparticles within the gate oxide of metal-oxide semiconductor field effect transistors (MOSFETs) were demonstrated to have potential for superior integration possibilities and storage characteristics as compared to continuous floating gate memories. ${ }^{1}$ The nanoparticles in these memories act as charge storage elements within the gate oxide. Nonvolatile memory write operation is accomplished by injecting charge into the nanoparticles by tunneling from the channel to alter the threshold voltage of this transistor. In a normal write/read/erase cycle, information is written by charge injection, read by measuring the subthreshold current-voltage characteristic, and erased by tunneling charge back into the channel. A single electron or hole stored on each nanoparticle in an array with a nanoparticle density of $3-10 \times 10^{11} \mathrm{~cm}^{-2}$ results in a threshold voltage shift of $0.3-0.5 \mathrm{~V}$ that is easily detected at room temperature, ${ }^{1,2}$ although this threshold voltage shift also depends upon the capacitor network, i.e., tunnel and top oxide thicknesses. It is believed that silicon nanoparticle floating gate memories could outperform conventional floating gate memory devices with faster read and write times, higher reliability, and lower power dissipation. However, unless the nanoparticles are uniform in size, density, and distance from the channel, and are free from deleterious interface states, charge transit times, charging voltages, threshold shifts may vary from one nanoparticle to another and limit switching speed.

Nanoparticle floating gate devices were fabricated previously in two ways: ${ }^{1}(i)$ ion implantation of excess silicon into the gate oxide; and (ii) direct chemical vapor deposition of nanometer-scale silicon islands onto the thermal oxide in the floating gate region. Silicon nanoparticles have also been generated as an aerosol by pyrolysis of silane $^{3}$ or disilane ${ }^{4}$ or by thermal evaporation of $\mathrm{Si}^{5}{ }^{5}$ In studies of $\mathrm{Si}$ nanoparticle luminescence, Brus and co-workers passivated the surface of Si nanoparticles by passing the aerosol and oxygen through a furnace to form a thermal oxide coating, or by oxidation of the $\mathrm{Si}$ nanoparticles after collecting them in a liquid to form a colloid. In this paper, we describe the first fabrication of continuous floating

* Electrochemical Society Active Member. gate devices in which the Si nanoparticles that are synthesized and passivated as an aerosol are directly incorporated into the floating gate.

Aerosol and other particles are commonly regarded as anathema to microelectronic device fabrication. Not only do particles create defects that lead directly to device failure, they may also transport contaminants such as transition metals that degrade performance. We seek to produce near-monolayer coverage of the gate region with nanoparticles that are formed in a high temperature aerosol reactor. A monolayer is defined as the number of particles required to produce a single hexagonal close pack particle layer. The high temperature reactive atmosphere could lead to evaporation of such contaminants from surfaces in the reactor. The high surface area of the nanoparticles, approximately seven times the wafer area at monolayer coverage by uniformly sized particles, increases the risk of contamination since the particles will effectively getter the contaminant vapors as the aerosol cools. An aerosol process can only be incorporated into device fabrication if the contamination issues can be addressed.

This paper describes an aerosol synthesis and deposition tool that meets the stringent cleanliness requirements of a modern ultralargescale integration (ULSI) microelectronic fabrication facility. Surface contamination of transition metals, e.g., $\mathrm{Fe}, \mathrm{Cr}$, and $\mathrm{Zn}$, and $\mathrm{Li}, \mathrm{Na}$, $\mathrm{K}$, and $\mathrm{Al}$ have been kept below or close to $10^{10}$ atoms $\mathrm{cm}^{-2}$ while depositing approximately $10^{12} \mathrm{Si}$ nanoparticles $\mathrm{cm}^{-2}$ on the surface of $200 \mathrm{~mm}$ wafers, enabling production of aerosol nanoparticle discontinuous floating gate devices with novel properties.

\section{Experimental}

Gas delivery system.-The ultraclean two-stage aerosol reactor system is illustrated in Fig. 1. Electronic grade gases $(0.5 \%$ silane $\left(\mathrm{SiH}_{4}\right)$ in nitrogen, nitrogen, and oxygen) with a minimum $99.999 \%$ purity were used. To remove trace amounts of oxygen from the pyrolysis furnace upstream of the oxidation furnace, a Nanochem PuriFilter PF-25 was placed after the gas regulator on the nitrogen stream. Mass flow controllers with VCR connections control the 1500 standard cubic centimeters per minute $(\mathrm{sccm})$ nitrogen and variable oxygen flows. An ultraclean metering valve was used to control the very small $(<1 \mathrm{sccm})$ silane flow rate. 0.25 in. 316 stainless steel tubing was obtained with an electropolished finish maximum of 10 roughness average (R.A.). All connections were 


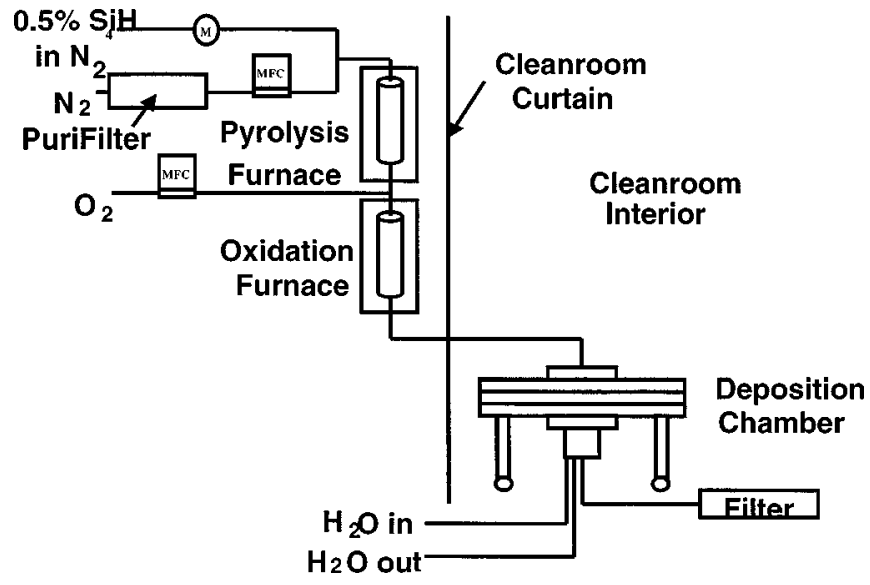

Figure 1. Schematic of the ultraclean reactor and deposition chamber. The deposition chamber is housed within a class 100 cleanroom.

made with VCR fittings with an electropolished finish of 7-10 R.A. Straight pieces of 0.25 in. 316 stainless steel tubing were cut and VCR glands were welded in an ultrahigh pure argon environment. All pieces were then electropolished and passivated to an R.A. of 7-10. Any bends in the system were made with VCR elbows, not by bending the electropolished tubing.

Pyrolysis and oxidation furnaces. - The $\mathrm{SiH}_{4}$ pyrolysis tube furnace operates at $900^{\circ} \mathrm{C}$ with a total heated length of $20 \mathrm{~cm}$. The oxidation tube furnace operates at $1050^{\circ} \mathrm{C}$ with a total heated length of $36 \mathrm{~cm}$. Given typical flow rates of gases through both furnaces (1.5 $\mathrm{L} \mathrm{min}^{-1}$ for pyrolysis and $1.75 \mathrm{~L} \mathrm{~min}^{-1}$ for oxidation), a 19/17 $\mathrm{mm}$ quartz tube gives a residence time of $\sim 3 \mathrm{~s}$ in each furnace. An hour prior to making silicon nanoparticle samples, the nitrogen flow is turned on so that any residual oxygen in the system is removed. $\mathrm{No} \mathrm{SiH}_{4}$ is introduced into the system until the temperature of the pyrolysis furnace reaches $900^{\circ} \mathrm{C}$ to insure complete reaction of $\mathrm{SiH}_{4}$.

Electronic grade quartz tubing $(19 / 17 \mathrm{~mm})$ was treated with an RCA clean (a recipe developed by the RCA Company) prior to installation in each furnace. For the RCA clean, all liquid reagents used were minimum $99.9 \%$ purity. For the RCA clean, the quartz tube was immersed in $18 \mathrm{M} \Omega \mathrm{H}_{2} \mathrm{O}: 30 \% \mathrm{H}_{2} \mathrm{O}_{2}: \mathrm{NH}_{4} \mathrm{OH}$ in the volume ratio of $5: 1: 1$ at $80^{\circ} \mathrm{C}$ for $20 \mathrm{~min}$. The tube was then rinsed with $18 \mathrm{M} \Omega \mathrm{H}_{2} \mathrm{O}$. To remove heavy metals, the tube was then immersed in $18 \mathrm{M} \Omega \mathrm{H}_{2} \mathrm{O}: 30 \% \mathrm{H}_{2} \mathrm{O}_{2}: \mathrm{HCl}$ in the volume ratio of $6: 1: 1$ at $80^{\circ} \mathrm{C}$ for $20 \mathrm{~min}$. Again, the tube was rinsed with $18 \mathrm{M} \Omega \mathrm{H}_{2} \mathrm{O}$. At all steps, great care was taken to avoid touching the quartz with ungloved hands. The quartz tubes were installed in the reactor system with electropolished UltraTorr fittings (R.A. 7-10) that interfaced to VCR fittings.

Deposition chamber.-The deposition chamber is constructed from 316 stainless steel vacuum flanges as shown schematically in Fig. 2. This chamber is radially symmetric. The aerosol flow enters the top of the deposition chamber along its centerline. The gas velocity decreases as it moves radially outward along the water surface. The gas then exits the chamber below the wafer at the center of the deposition chamber. The particles deposit on the top of the cool wafer by thermophoresis. The top of the deposition chamber is heated to $200^{\circ} \mathrm{C}$ and the water cooled disk in the interior of the chamber is maintained at $23^{\circ} \mathrm{C}$ with a cooling water supply. Since the particles migrate by thermal diffusion from high temperatures toward lower ones, the particles deposit on the silicon wafer. On the bottom side of the 9 in. diam water cooled disk (away from the aerosol inlet), 0.12 in. copper tubing encased in a stainless steel shell is wound around the disk to maximize heat exchange. Room temperature water flows through this 0.12 in. copper tubing and serves

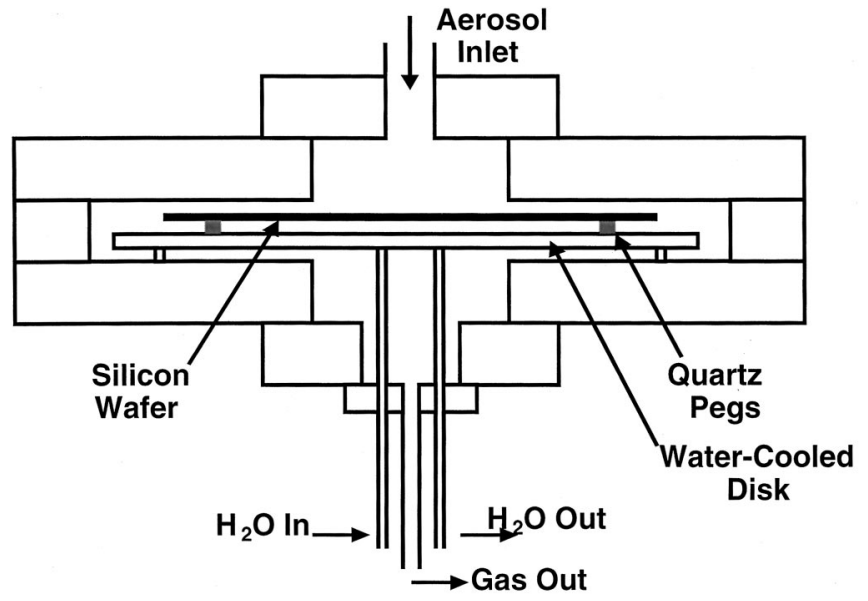

Figure 2. Schematic of the ultraclean deposition chamber. This chamber allows controlled thermophoretic deposition of nanoparticles onto a $200 \mathrm{~mm}$ silicon wafer.

to maintain a temperature gradient within the deposition chamber. To eliminate any contact between the metallic deposition chamber and the silicon wafer, the silicon wafer is supported by three quartz pegs that have been RCA cleaned using the same procedure used to clean the furnace quartz tubes. The quartz pegs rest on the top surface of the water cooled disk. Only a small contact area is made between these three quartz pegs and the silicon wafer. The interior surfaces of the deposition chamber and the water cooled disk were electropolished to an R.A. of 7-10 prior to use.

Class 100 cleanroom.-A modular class 100 cleanroom is used to store wafers and to house the deposition chamber. The two-stage aerosol reactor is located outside of the cleanroom. The 0.25 in. stainless steel tube that connects the reactor to the deposition chamber passes through a small hole in the cleanroom wall.

TXRF/SIMS.-Total reflection X-ray fluorescence (TXRF) analysis was performed with a TREX 610-T TXRF instrument. Surface secondary ion mass spectrometry (SIMS) analysis was performed with a Cameca IMS. Prior to building the cleanroom, ultraclean reactor and deposition chamber, TXRF analysis was performed on a wafer sample with silicon nanoparticles deposited on the surface. For this sample, the substrate and particles were exposed to normal laboratory operating conditions. Great care was taken to insure that the sample would be as clean as possible. Once the cleanroom and ultraclean reactor and deposition chamber were built, wafer samples were prepared for TXRF/SIMS analysis. A cleanroom sample was prepared by exposing a silicon wafer to the cleanroom environment for $5 \mathrm{~min}$. In preparing this sample, the wafer was removed from the wafer box, carried to and from the deposition chamber with a quartz fork without putting the wafer in the deposition chamber. The wafer was then replaced in the wafer box. This procedure was used to simulate the normal transport of a wafer around the cleanroom prior to and following sample preparation. Two separate wafers were put into the deposition chamber and particles were deposited on them. In these experiments, the reactor was operating at the normal conditions expected for sample collection. Separate samples were collected with the oxidation furnace off and with it turned on. Particles were deposited on the wafer surface for a total collection time five times longer than executed for nanoparticle device fabrication, providing a worst-case scenario. Dense nanoparticle deposits were readily visible on the wafer surfaces.

Experimental AFM measurements.-In order to verify the particle deposition, two separate aerosol samples were collected by changing the gap spacing in the deposition chamber. This was done by changing the size of the quartz pegs supporting the silicon wafer. 


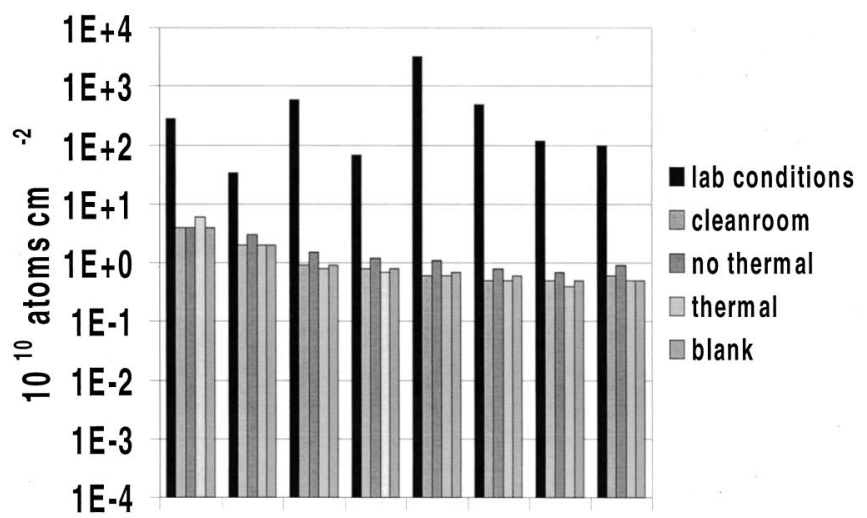

(a)
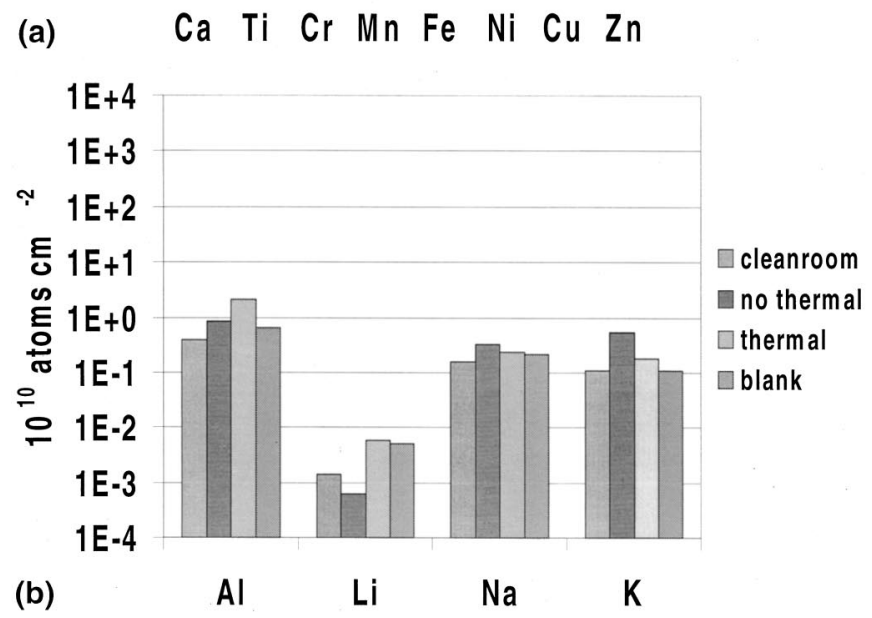

Figure 3. TXRF/SIMS data (a) TXRF data from five different samples. The sample made under normal lab conditions has unacceptably high levels of contamination. All samples made in the ultraclean reactor and deposition chamber have acceptable low levels of contamination. (b) SIMS data from four samples. All samples made in the ultraclean reactor and deposition chamber have acceptable levels of contamination.

After each specified collection time, the wafers were removed from the deposition chamber and the particle densities were measured with an atomic force microscope (AFM). Using the AFM, a radial particle density profile was obtained. Ten measurements were taken at $1 \mathrm{~cm}$ intervals from the edge of the wafer toward the wafer center. The resulting AFM images were used to estimate particle densities by counting the size and number of particles on each image. Although the AFM can not accurately measure the lateral dimensions of the particles, the particle heights are accurate, enabling calculations of the particle size and density.

\section{Results}

TXRF/SIMS.-The TXRF results appear in Fig. 3a. The "lab conditions" sample was prepared prior to building the ultraclean reactor and deposition chamber. Since the sample showed unacceptably high levels of contamination, the necessity for building the ultraclean reactor, deposition chamber, and cleanroom became apparent. The "blank" sample was considered to be free of any contamination. No measurable contamination was detected on the cleanroom sample. The two wafers made in the ultraclean reactor and deposition chamber clearly demonstrates dramatic decreases in the levels of contamination measured by TXRF. SIMS measurements of $\mathrm{Al}, \mathrm{K}, \mathrm{Na}$, and $\mathrm{Li}$ of the two wafers produced in the ultraclean reactor and the cleanroom exposed wafer are presented in Fig. $3 \mathrm{~b}$ along with the blank sample. Again, no significant contamination was detected in the samples handled in the ultraclean reactor and deposition chamber. When analyzing TXRF and SIMS data, any reported values within a factor of 2 to the values measured on the

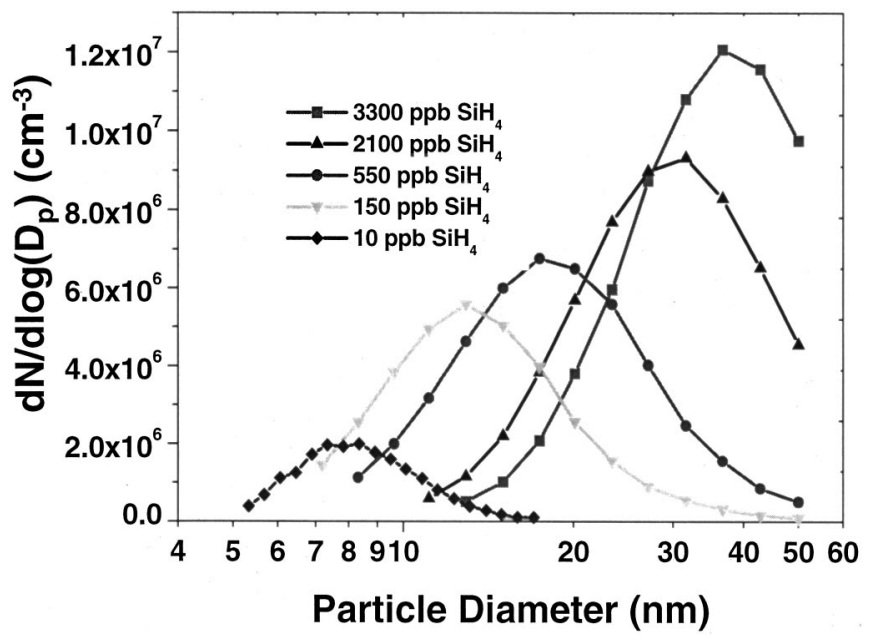

Figure 4. The silane concentration in the aerosol reactor determines the nanoparticle size distribution. By decreasing the silane concentration, smaller particles are produced.

blank are regarded as the upper limits of the actual contamination. Thus, a wafer is still considered to be contamination free if its measured surface concentration is a factor of 2 larger than the blank wafer. Since no contamination levels greater than $10^{11}$ atoms $\mathrm{cm}^{-2}$ were detected, the system is operating within acceptable contamination levels.

Particle size distribution.-The particle size distribution has been controlled by varying the silane concentration in the two-stage aerosol reactor. Figure 4 shows the resulting variation in the particle size distribution with silane concentration of thermal oxide passivated silicon nanoparticles produced in the two-stage aerosol reactor. Size distributions have been measured using a radial differential mobility analyzer ${ }^{6,7}$ with an electrometer as a detector. The resulting particle size distributions of thermal oxide-passivated silicon nanoparticles from the ultraclean reactor vary by about $50 \%$ in diameter. By decreasing the silane concentration in the reactor, the particle size distribution shifts to smaller particle sizes. At a given silane concentration, the resulting particle size distribution is extremely stable over time.

Particle structure.-Extensive work has been done by the authors to produce uniformly sized, single crystal $\mathrm{Si} / \mathrm{SiO}_{2}$ nanoparticles for applications in nonvolatile memory devices. The two-stage aerosol reactor is capable of making high quality particles that are dense, spherical, nonagglomerated single crystals. Samples of thermal oxide passivated silicon nanoparticles were collected on holey carbon transmission electron microscope grids and can be seen in Fig. 5. The spherical single crystal particles are between 3 and $7 \mathrm{~nm}$ in size as represented by the $10 \mathrm{ppb} \mathrm{SiH}_{4}$ curve in Fig. 4. Additionally, larger particles with crystalline cores of approximately $8 \mathrm{~nm}$ can be seen in Fig. 6 .

Particle deposition and chamber modeling.-In order to verify that particles could actually be deposited using this deposition chamber, the deposition chamber was modeled using the commercial computational fluid dynamics code FLUENT. Experimental verification of particle deposition was obtained by using an AFM. FLUENT has been used to calculate velocity streamlines and temperature contours. Particle trajectories were also calculated by modifying FLUENT to include equations that describe the behavior and velocity of particles in a temperature gradient. These particle trajectories were used to estimate the uniformity of particle deposits and to understand design improvements that could be implemented in the future to obtain more uniform deposits. This model has also been used to estimate radial densities of particle deposits. This model 


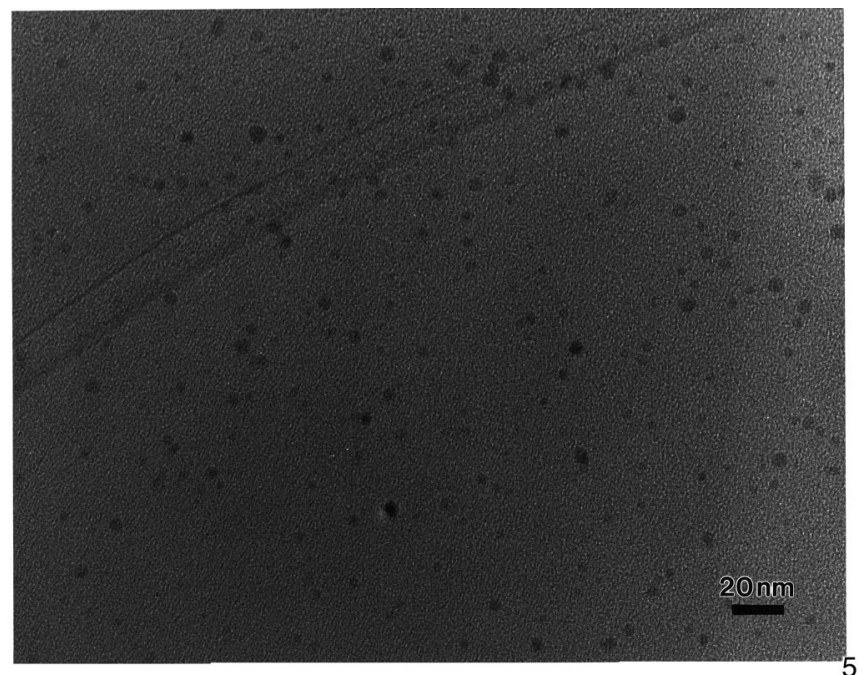

Figure 5. TEM image of single crystal $\mathrm{Si} / \mathrm{SiO}_{2}$ nanoparticles collected on holey carbon that are 3-7 $\mathrm{nm}$ diam spheres.

allows one to vary the inlet aerosol flow rate, the temperature of the heated deposition chamber top, the temperature of the water cooled disk, and the gap spacing between the inside of the top deposition chamber and the wafer surface.

Figure 7 shows calculated streamlines, temperatures, and particle trajectories for the conditions used in device fabrication. As seen in the velocity streamlines (Fig. 7a), the abrupt expansion in the flow cross section near the entrance to the deposition chamber (top) leads to a large recirculation region. Most of the incoming flow remains in a well defined jet near the centerline until it approaches the wafer where it forms a radial outward wall layer flow. The flow deviates from the wafer surface near the edge of the cavity, but quickly reestablishes the radial flow in the outer regions. Below the wafer and the cooling plate, the flow is drawn radially inward toward the central outlet port.

The temperature contours (Fig. 7b) are also strongly influenced by the recirculation within the entrance cavity. The temperature gradient is high in the central jet impingement region, decreases near

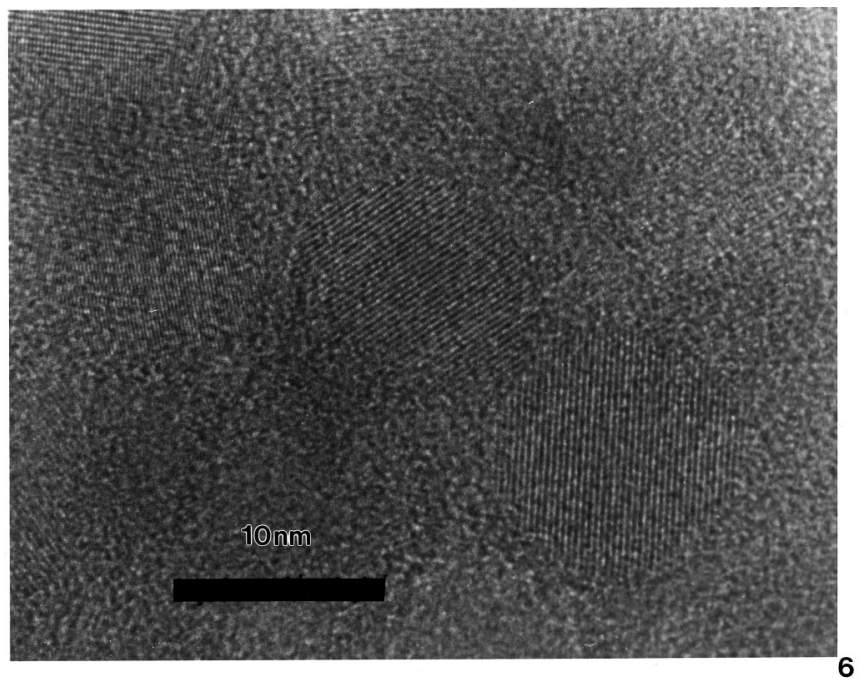

Figure 6. High resolution TEM image of $8 \mathrm{~nm}$ single crystal particles formed in the ultraclean reactor. This image demonstrates that the crystalline particles are not completely oxidized in the oxidation furnace.
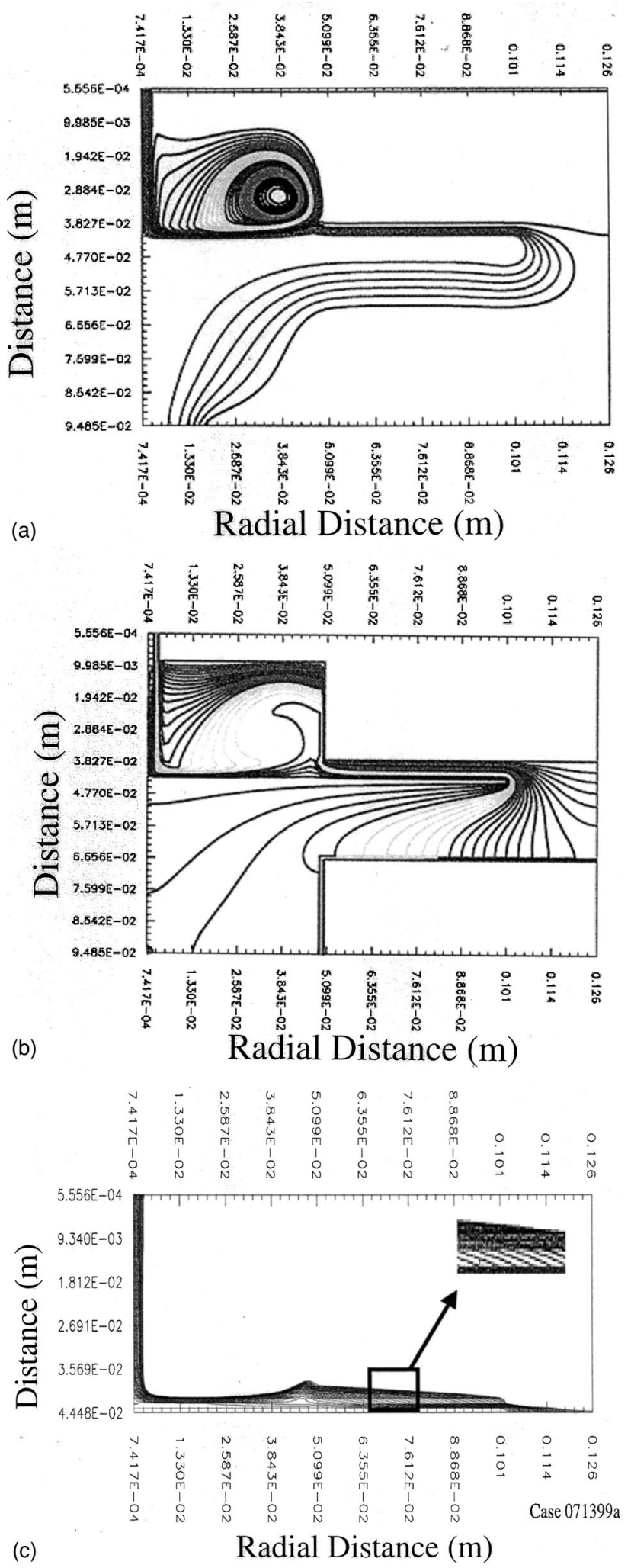

Figure 7. Results of FLUENT simulations (a) Velocity streamlines illustrate the recirculation area in the entrance of the deposition chamber. The velocity streamlines become very uniform along the outer edges of the deposition chamber. (b) Temperature contours exhibit nonuniformities in the large entrance cavity that stabilize in regions where the wafer and chamber gap become very uniform. (c) Particle trajectories are calculated by combining the velocity streamlines and temperature contours together with particle behavior in temperature gradients. The inset illustrates the particle trajectories as they contact the silicon wafer surface. 


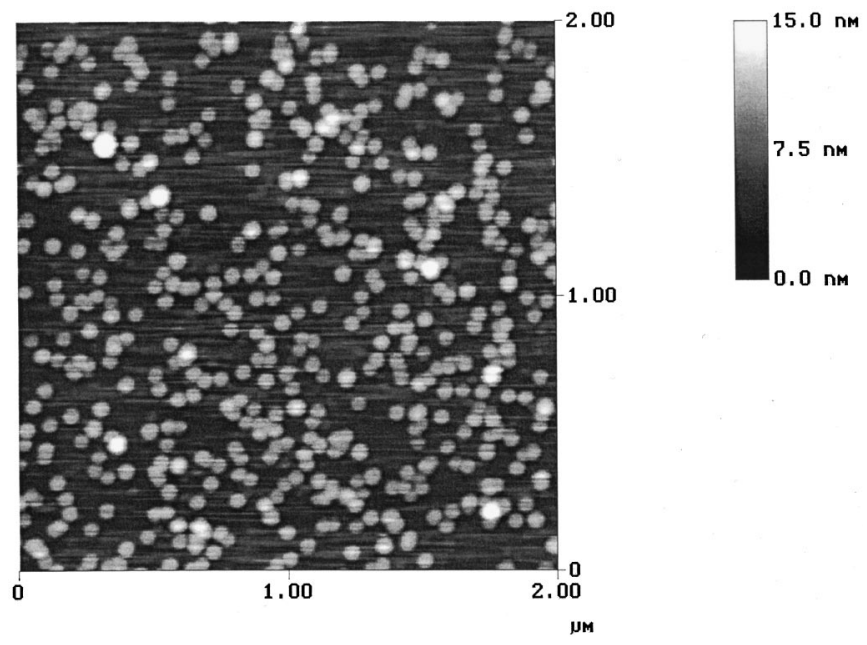

Figure 8. Representative AFM image taken on a wafer with deposited nanoparticles. Such an image was taken every centimeter from the wafer edge toward the wafer center. These images were then used to estimate particle coverages on the wafer.

the cavity edge, and then increases in the outer region where the flow is confined to the small gap between the wafer and the top of the chamber.

Particle trajectories (Fig. 7c) follow the incoming gas streamlines but the particles are affected by the temperature gradient within the deposition chamber. To account for these thermophoretic forces, an additional component to the particle velocity, the thermophoretic velocity $v_{\mathrm{t}}$, was calculated using the expression given by Flagan and Seinfeld ${ }^{8}$

$$
v_{\mathrm{t}}=\frac{-T h \mu \frac{d T}{d x}}{\rho T}
$$

Th is the thermal dimensionless group and is assumed to be 0.5 for nanoparticles; $\mu, \rho$, and $T$ are the viscosity, density, and temperature of the carrier gas (nitrogen in this study). As seen from the equation, as the temperature gradient $(d T / d x)$ increases, the thermophoretic velocity also increases.

AFM measurements.-In order to verify that particles are being deposited on the silicon wafers and to test the deposition chamber model, two different wafer samples were collected. The particle densities were estimated from AFM images. A typical example is shown in Fig. 8. The estimated radial particle density profile calculated from AFM images for the two samples is seen in Fig. 9. In general, the outer edges of the wafer show very uniform particle deposits as the model predicts. Toward the center of the wafer, a large increase in the particle density is observed. The wafer center had the highest concentration of particle deposits on the entire wafer where particles are depositing because of stagnation flow. The nonuniform nanoparticle densities collected on each wafer is currently a tremendous advantage. With a single silicon wafer, we can produce devices that have nanoparticle densities ranging between 10 and $100 \%$ monolayer. Thus, we do not need to prepare separate wafer samples to determine the effect of particle density on memory performance. All density effects can be probed on the same wafer simply by moving to a different radial position. However, once nanoparticle density effects on memory performance are known, future devices will require uniform particle densities over larger areas of the wafer. Thus, a new deposition chamber will then be required.

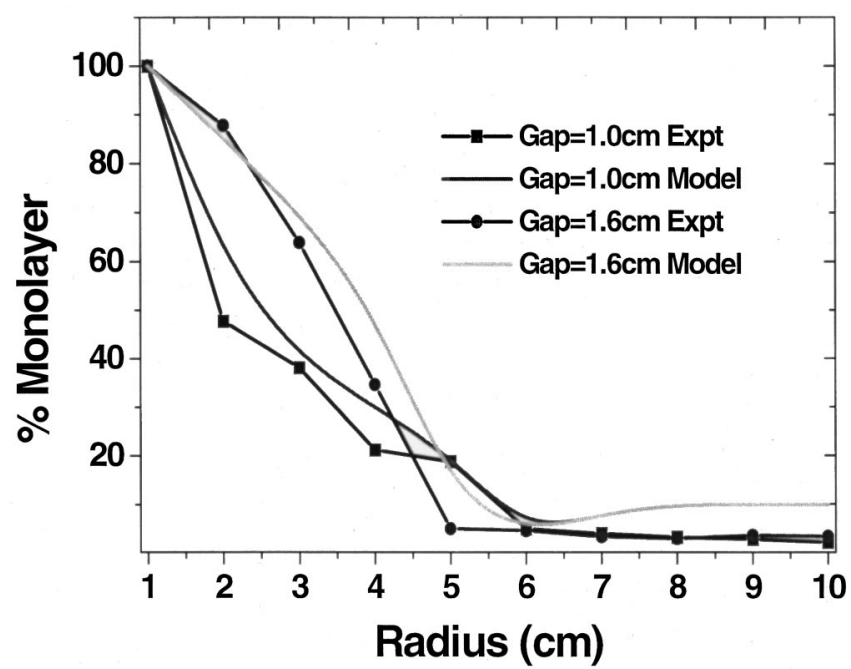

Figure 9. Nanoparticle density as a function of wafer radius. The experimental densities were estimated from AFM images such as those in Fig. 8 taken of two separate wafers at gap distances of 1.0 and $1.6 \mathrm{~cm}$. The gap corresponds to the distance between the silicon wafer surface and the bottom of the top plate of the deposition chamber. This gap is varied by using different quartz peg lengths.

\section{Discussion}

We have designed an ultraclean two-stage aerosol process reactor and $200 \mathrm{~mm}$ wafer deposition chamber that is currently being used to integrate $\mathrm{Si} / \mathrm{SiO}_{2}$ nanoparticles into memory devices. Silicon nanoparticles are synthesized by thermal decomposition of silane gas and are passivated with thermal oxide. This two-stage aerosol reactor has been successfully integrated with a $200 \mathrm{~mm}$ silicon wafer deposition chamber that is contained within a class 100 cleanroom. This entire reactor system conforms to rigorous cleanliness specifications such that we can control the contamination of transition metals to levels below or close to $10^{10}$ atoms $\mathrm{cm}^{-2}$ while still obtaining particle deposition densities of $10^{12}$ particles $\mathrm{cm}^{-2}$. The deposition chamber has been designed to produce a controllable particle density profile along a $200 \mathrm{~mm}$ wafer where particles are thermophoretically deposited uniformly over three-quarters of the wafer area. Thus, we now have the capability to deposit controlled densities of oxide-passivated silicon nanoparticles onto 8 in. silicon wafers for production of silicon nanoparticle memory devices.

Improvements for the future include designing a deposition chamber that produces uniform deposits over the entire wafer surface. Once current memory devices have been experimentally tested and analyzed, appropriate nanoparticle densities for optimal memory performance will be known. The flow model of which the accuracy has been experimentally determined will be used to identify suitable reactor designs. At that point, nanoparticle wafers will be produced that have constant and optimal nanoparticle densities over the entire wafer. To improve the particle size distribution, we eventually hope to add an ultraclean differential mobility analyzer to size classify the particles prior to deposition to improve control over the particle size distribution beyond that attained with the present ultraclean reactor.

\section{Conclusions}

Preliminary characterization of device fabrication using the present reactor suggest that the aerosol nanoparticle floating gate devices exhibit normal transistor behavior and have very promising nonvolatile device performance. Aerosol nanoparticle devices with $0.2 \mu \mathrm{m}$ channel length exhibited large threshold voltage shifts $(>3$ $\mathrm{V})$, submicrosecond program times, and millisecond erase times. No degradation in program/erase threshold voltage swing was seen during 100,000 program and erase cycles, although some threshold shift 
due to charge trapping was observed. Retention times greater than $50,000 \mathrm{~s}$ have been observed to date for these devices. Details will follow in a separate paper.

\section{Acknowledgment}

This work was supported by NSF grant no. DMR-9871850.

The California Institute of Technology assisted in meeting the publication costs of this article.

\section{References}

1. S. Tiwari, F. Rana, K. Chan, K. Shi, and H. I. Hanafi, Appl. Phys. Lett., 69, 1232 (1996).

2. H. I. Hanafi, S. Tiwari, and I. Khan, IEEE Trans. Electron Devices, 43, 1553 (1996).

3. J. J. Wu, H. V. Nguyen, and R. C. Flagan, Langmuir, 3, 266 (1987).

4. K. A. Littau, P. J. Szajowski, A. J. Muller, A. R. Kortan, and L. E. Brus, J. Phys. Chem., 97, 1224 (1993).

5. R. P. Camata, H. A. Atwater, and R. C. Flagan, Appl. Phys. Lett., 68, 3162 (1996).

6. S. H. Zhang, Y. Akutsu, L. M. Russell, R. C. Flagan, and J. H. Seinfeld, Aerosol. Sci. Technol., 23, 357 (1995).

7. S. H. Zhang and R. C. Flagan, J. Aerosol. Sci., 27, 1179 (1996).

8. R. C. Flagan and J. H. Seinfeld, Fundamentals of Air Pollution Engineering, p. 313, Prentice Hall, New York (1988). 